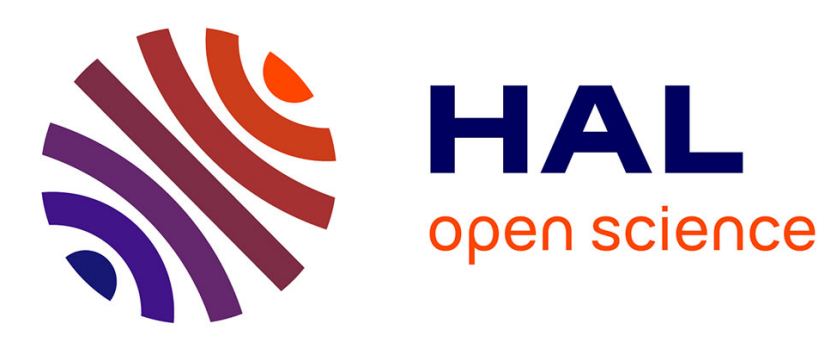

\title{
Une méthode mécanique pour mesurer la pression de vapeur d'équilibre de l'eau dans un milieu complexe
}

\author{
Samuel Ouoba, Bruno Cousin, Fabien Cherblanc, Jean-Claude Benet
}

\section{To cite this version:}

Samuel Ouoba, Bruno Cousin, Fabien Cherblanc, Jean-Claude Benet. Une méthode mécanique pour mesurer la pression de vapeur d'équilibre de l'eau dans un milieu complexe. Comptes Rendus Mécanique, 2010, 338, pp.113-119. 10.1016/j.crme.2010.02.005 . hal-00532806

\section{HAL Id: hal-00532806 https://hal.science/hal-00532806}

Submitted on 7 Jun 2011

HAL is a multi-disciplinary open access archive for the deposit and dissemination of scientific research documents, whether they are published or not. The documents may come from teaching and research institutions in France or abroad, or from public or private research centers.
L'archive ouverte pluridisciplinaire HAL, est destinée au dépôt et à la diffusion de documents scientifiques de niveau recherche, publiés ou non, émanant des établissements d'enseignement et de recherche français ou étrangers, des laboratoires publics ou privés. 


\title{
A new experimental method to determine the
}

\section{sorption isotherm of a liquid in a porous medium}

\author{
Samuel Ouoba, Fabien Cherblanc *, Bruno Cousin, and Jean-Claude Bénet \\ E-mail: Fabien.Cherblanc@univ-montp2.fr \\ Tel: (+33) 467-149-639 - Fax: (+33) 467-149-639 \\ Laboratoire de Mécanique et Génie Civil, CNRS, Université Montpellier 2, Place Eugène \\ Bataillon, 34095 Montpellier cedex 5, France \\ E-mail:
}

\begin{abstract}
Sorption from the vapor phase is an important factor controlling the transport of volatile organic compounds (VOCs) in the vadose zone. Therefore, an accurate description of sorption behavior is essential to predict the ultimate fate of contaminants. Several measurement techniques are available in the case of water, however, when dealing with VOCs, the determination of sorption characteristics generally relies on gas chromatography. To avoid some drawbacks associated with this technology, we propose a new method to determine the sorption isotherm of any liquid compounds adsorbed in a soil. This method is based on standard and costless transducers (gas pressure, temperature) leading to a simple and transportable experimental device. A numerical estimation underlines the good accuracy and this technique is validated on two examples. Eventually, this method is applied to determine the sorption isotherm of three liquid compounds (water, heptane and trichloroethylene) in a clayey soil.
\end{abstract}

\footnotetext{
*To whom correspondence should be addressed
} 


\section{Introduction}

Porous media such as soils, agro-products or building manufactured materials, generally contain a liquid phase in the form of adsorbed layers on the solid phase surface. These adsorbed layers are sufficiently thin to be sensitive to small range interactions like Van der Waals forces. Therefore, the thermodynamic state of the adsorbed liquid phase deviates from normal conditions ( 1 ). These deviations manifest themselves at the macroscopic scale through the sorption behavior meaning that the liquid activity, $a_{l}$, is lower than 1 . The upper limit, $a_{l}=1$, corresponds to the pure liquid compound. Thus, the liquid activity relates to the chemical potential and represents the energy required to extract the liquid compound from the porous medium. This characteristic is therefore directly involved in groundwater risk assessment.

Hygroscopicity generally refers to an adsorbed water phase. It governs, for instance, the dessication process of subsurface galleries drilled in deep waste deposit projects. However, these effects are also observed with any liquid phase such as hydrocarbons or volatile organic compounds (VOCs). Sorption processes are widely acknowledged to play an important role in the transport and fate of VOCs in subsurface systems (2-5). Therefore, contaminant sorption is a determining characteristic to choose the appropriate remediation technique to be carried out in sites polluted by hazardous substances. Concerning the soil vapor extraction technology, the low recovery period that often last for years is partially linked to the high sorptive capacity of soils. To propose accurate predictions of contaminant sorption, many theoretical models have been developed. However, their appropriateness to a contaminated site relies on the experimental technique able to characterize a natural soil sample.

The most common methods are only dedicated to water sorption characterization. Since the water activity is also equal to the equilibrium relative humidity, $R H$, water sorption behavior is generally established using standard saturated salt solutions. This method provides accurate results, nevertheless, the time required to reach equilibrium can be large ( $\approx 1$ month). Some attempts to decrease measurement times by modeling the vapor diffusion process inside the sample have been proposed (6). 
Recently, automated devices have been developed to establish the sorption isotherm curve faster. For instance, with the Dynamic Vapor Sorption (DVS) method (7), a sample is subjected to varying conditions of humidity and temperature by using mass flow controllers, one for dry air and the other for air saturated with vapor. The response of the sample is measured gravimetrically. Devices are programmed to automatically cycle between different humidity constant at each level until equilibrium is reached. Several commercial instruments are based on this method (Dynamic Vapor Sorption instrument from Surface Measurement Systems Ltd, IGAsorp from Hiden Analytical, SGA-100 Symmetric Gravimetric Analyzer from VTI Corporation, CI Sorp Water Sorption Analyzer from CI Electronics Ltd, VTI-SA from TA Instruments). The relative humidity of air is generally measured by a chilled mirror dewpoint sensor. With this technology a mirror is gradually cooled while a light emitting diode associated with a photodetector monitors the reflected light in order to detect when the vapor begins to condense. The dewpoint temperature is directly related to the relative humidity by means of the saturated vapor pressure curve. Using this method, the Dynamic Dewpoint Isotherm (DDI) method continuously measures the water activity and water content without waiting for equilibration (Aqualab 4TE Duo from Decagon Devices Inc.) (8).

To deal with volatile organic compounds, some of the apparatus enumerated above are extended. Indeed, mass flow controllers can generate a variably vapor saturated atmosphere by mixing dry and vapor saturated air fluxes. However, the vapor pressure cannot be regulated since usual humidity sensors cannot be fitted to any organic compounds.

In the framework of surface area and pore size characterization, volumetric sorption analyzers have been developed (HTP1 from Hiden Analytical, BET Instruments from Micromeritics, Autosorb from Quantachrome Instruments). An inert gas is admitted around the sample in controlled increments. After each dose of adsorptive, the pressure is allowed to equilibrate and the quantity of gas adsorbed is calculated. The most commonly used adsorptive gas is nitrogen and these experimental devices are not designed to analyze any other vapor compound. An alternative is to use more sophisticated analyze techniques such as gas chromatography $(4,5,9-14)$. Nevertheless, these methods are much more circuitous and expensive to implement. 
To avoid the drawbacks associated with the methods presented above, we propose a new experimental method to determine the activity of a liquid adsorbed in a porous medium. This paper aims to characterize the uncertainties associated with this method, on one hand, from numerical estimation and, on the other hand, based on a comparison with usual experimental techniques. To assess the usefulness, sorption isotherms regarding to various liquid compounds (water, heptane, trichloroethylene) are presented.

\section{Material and Methods}

\section{Theoretical background}

The sorption isotherm curve gives the relation between the liquid activity, $a_{l}$, and the mass liquid content, $w_{l}$. The liquid activity, $a_{l}$, is related to the mass chemical potential of the liquid, $\mu_{l}$, by:

$$
\mu_{l}=\mu_{0}+\frac{R T}{M} \ln \left(a_{l}\right)
$$

where, $\mu_{0}$, is a reference chemical potential, $R$, is the ideal gas constant, $T$, is the temperature and, $M$, is the molar mass of the compound. The liquid activity is generally obtained by relying on the Gibbs equilibrium principle which specifies that, at equilibrium, the chemical potential of a component is identical in every compartment in contact through semi-permeable membranes. Therefore, the chemical potential of the liquid can be evaluated through the chemical potential of the vapor surrounding the porous sample, given by the relative humidity, $R H$ :

$$
\mu_{v}=\mu_{0}+\frac{R T}{M} \ln \left(\frac{P_{v}}{P_{v s}}\right)=\mu_{0}+\frac{R T}{M} \ln (R H)
$$

where, $P_{v}$, is the vapor partial pressure and, $P_{v s}$, is the saturating vapor pressure. At equilibrium, the equality of the chemical potentials, Eq. (2) and Eq. (1), leads to the determination of the liquid 
activity by:

$$
a_{l}=R H_{e q}
$$

Therefore, the determination of one point of the sorption isotherm curve, $\left(w_{l} ; a_{l}\right)$, requires the measurement of the equilibrium vapor partial pressure, $P_{v e q}$, surrounding the sample. With this objective, the experimental principle is detailed below.

\section{Description and principle}

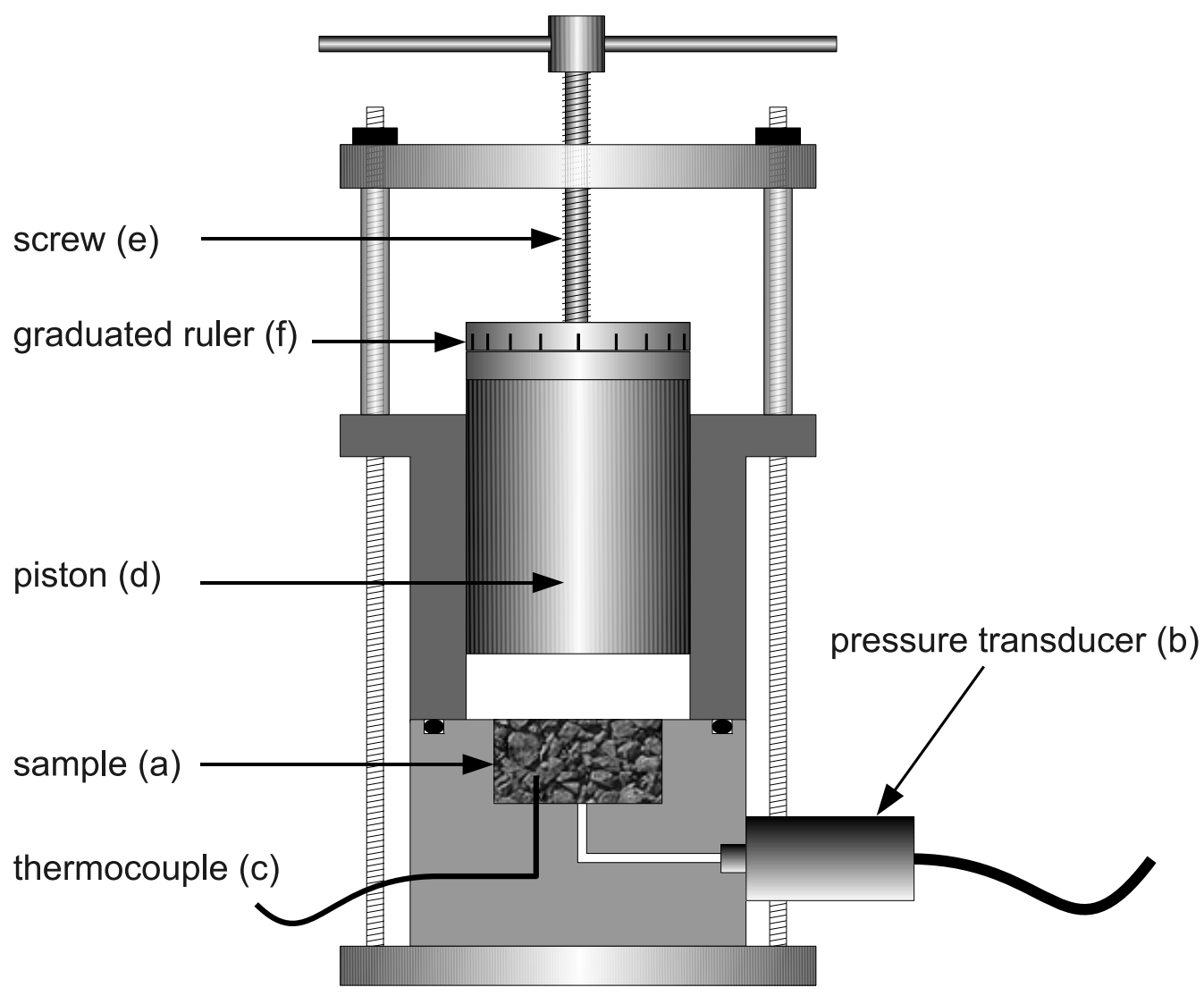

Figure 1: Schema of the activity-meter.

The experimental device, called "activity-meter", is schematized in Figure 1. This device and the associated method are protected by a patent applied for the French National Industrial Property Institute ( ${ }^{\circ} 09$ 05293) (15), commercial apparatus are supplied by TMI Orion SA, Montpellier, France. 
The sample (a) is disposed up against a pressure transducer (b) (Druck, PMP4030AB) and a temperature thermocouple (c) (type K). This allows us to record the total pressure of the gas phase, $P_{g}$, and its temperature, $T$, along the process. A piston pump (d) is placed above the sample in order to impose a gas pressure below the atmospheric pressure. The piston chamber volume is controlled by a screw system (e) with a graduated ruler (f). O-ring gaskets ensure a perfect airtightness so that the system can be considered thermodynamically closed. The dimensional characteristics of the device are given in Table 1. The whole device is placed in a thermo-regulated bath to ensure a constant temperature. Since the device is made of stainless steel, its large thermal inertia leads to weak temperature variations and thermal equilibrium is experimentally observed. Data acquisition is performed through a National Instrument DAQ card and analyzed using LabView.

Table 1: Dimensional characteristics of the experimental device.

\begin{tabular}{ll}
\hline Sample diameter & $32 \mathrm{~mm}$ \\
\hline Sample height & $15 \mathrm{~mm}$ \\
\hline Sample volume & $12.07 \mathrm{~cm}^{3}$ \\
\hline Piston diameter & $44.5 \mathrm{~mm}$ \\
\hline Piston displacement for one rotation & $1.75 \mathrm{~mm}$ \\
\hline Volume increment for one rotation & $2.722 \mathrm{~cm}^{3}$ \\
\hline
\end{tabular}

The gas phase enclosed in the experimental device is composed of dry air and of the vapor of the compound adsorbed in the porous sample. In standing conditions, the vapor is in equilibrium with the liquid meaning that its partial pressure is equal to its equilibrium vapor pressure, $P_{v e q}$. Starting from an equilibrium situation, the principle of an experiment consists in moving up the piston to a new position by using the screw system and recording the evolution of the gas phase pressure and the temperature. After a while, the gas pressure tends to a constant value that defines a new equilibrium situation. This stage is repeated several times in order to provide a set of value triplets $\left(T^{i}, V^{i}, P_{g}^{i}\right) i \in\left[1 . . n_{S}\right]$, where $T^{i}$ is the measured equilibrium temperature, $V^{i}$ is the volume imposed by the piston, $P_{g}^{i}$ the measured equilibrium total gas pressure and $n_{s}$ is the number of equilibrium stages.

The temperature, $T$, and total gas pressure, $P_{g}$, recorded through the data card acquisition are schematized in Figure 2. This typical experiment is composed of 3 successive equilibrium 
stages $\left(n_{s}=3\right)$. Starting from the atmospheric pressure, the gas pressure decreases at each piston displacement. This activates water evaporation observed through the slow rise of gas pressure until equilibrium. A slight temperature drop is experimentally observed resulting from liquid-gas interface cooling while evaporation occurs. Nevertheless, the thermal regulation quickly ensures a constant and homogeneous temperature inside the device.

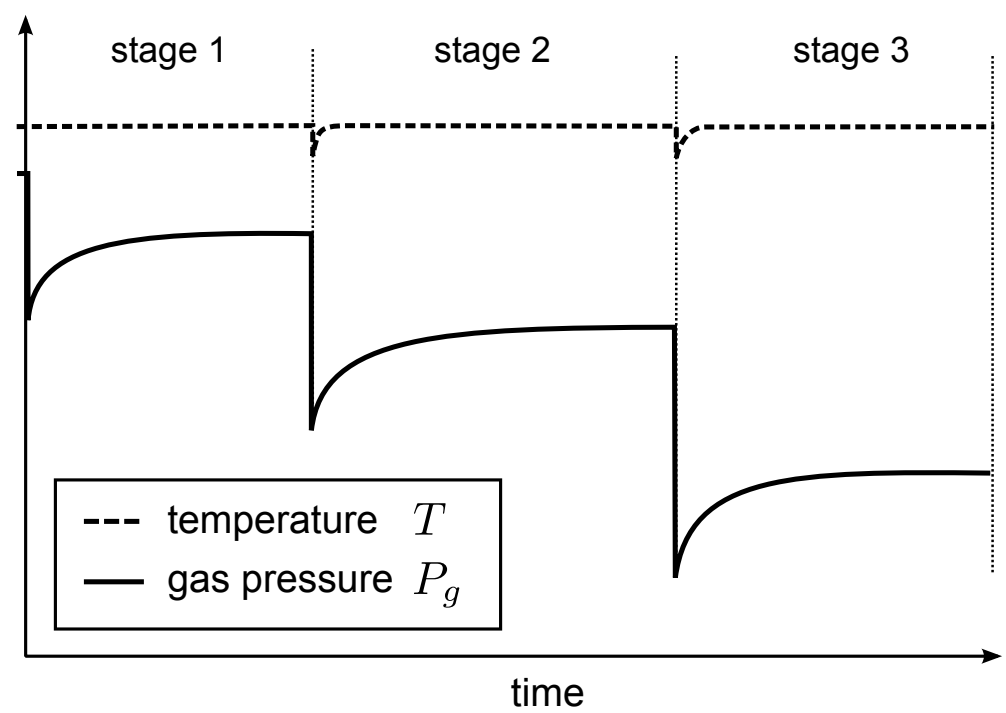

Figure 2: Schematic representation of temperature, $T$, and total gas pressure, $P_{g}$, evolutions during an experiment composed of 3 equilibrium stages.

Considering a sample at a given liquid content, $w_{l}$, the unknown physical quantities are:

- $V_{0}$, the gas phase volume in the initial equilibrium position. This includes the pore space inside the sample as well as the initial volume of the piston chamber and dead volumes in the pressure transducer;

- $n_{a}$, the mole number of the dry air inside the device that remains constant since it is a closed system;

- $P_{v e q}$, the equilibrium vapor pressure. Since the liquid amount evaporated during an experiment is negligible, the liquid content, $w_{l}$, is considered to be constant. This assumption has been experimentally checked by measuring the liquid content before and after each experiment. Therefore, the equilibrium vapor pressure, $P_{v e q}$, can also be considered constant. 
By assuming that dry air behaves as an ideal gas, the gas pressure can be written for each equilibrium stages as the sum of partial pressures of vapor and air:

$$
P_{g}^{i}=P_{v e q}+P_{a}=P_{v e q}+n_{a} \frac{R T^{i}}{V_{0}+V^{i}}
$$

The ideal gas description is legitimate since air is far from its liquid-gas phase transition. To determine the 3 unknown quantities described above, $\left(V_{0}, n_{a}, P_{v e q}\right), 3$ equilibrium stages are sufficient: $\left(T^{i}, V^{i}, P_{g}^{i}\right) i \in[1 . .3]$. However, to increase accuracy, 6 to 10 volume increments are performed. Thus, the 3 unknown are identified using a non-linear least-square minimization procedure based on the standard Levenberg-Marquadt algorithm (16).

With this procedure, the liquid activity is measured at pressures different from atmospheric conditions, 1 bar $<P_{g}<0.5$ bar. Actually, the liquid activity is not very sensitive to a pressure change; this can be assessed for water as follows $(17,18)$ :

$$
\ln \left(\frac{a_{w}^{2}}{a_{w}^{1}}\right)=\frac{M}{\rho_{w} R T}\left(P_{g}^{2}-P_{g}^{1}\right)
$$

For instance, the activity deviation can be overestimated by assuming a gas pressure variation of $P_{g}^{2}-P_{g}^{1}=10^{5} \mathrm{~Pa}$. This leads to $\Delta a_{w}=0.0007$, what can be considered negligible in this context.

\section{Uncertainties characterization}

With the experimental protocol described in the previous section, volume increments, $V^{i}$, are imposed while the total gas pressure, $P_{g}^{i}$, and the temperature, $T^{i}$, are measured. Associated with each of these three quantities, the uncertainties are:

- the temperature uncertainty of the thermocouple is estimated by, $\delta T= \pm 0.1 \mathrm{~K}$;

- the pressure accuracy is a characteristic of the pressure transducer given by the manufacturer (Druck, PMP4030AB), $\delta P= \pm 40 \mathrm{~Pa}$;

- the volume increment uncertainty mainly results from ruler reading (f). In terms of rotation 
angle, it can be easily less than $\delta \alpha= \pm 1.3^{\circ}$. Based on the characteristics of the device given in Table 1, this leads to $\delta V= \pm 0.01 \mathrm{~cm}^{3}$.

Taking into account these uncertainties, the total accuracy of the method is estimated numerically as presented below.

1. Based on a virtual ideal experiment, a series of triplets $\left(T^{i}, V^{i}, P_{g}^{i}\right) i \in\left[1 . . n_{s}\right]$ is computed using Eq. (4).

2. This theoretical experiment is modified using the uncertainties given above, i.e., random experimental points are generated inside the physical range ( $\left[T^{i}-\delta T, T^{i}+\delta T\right] ;\left[P_{g}^{i}-\delta P, P_{g}^{i}+\delta P\right]$; $\left.\left[V^{i}-\delta V, V^{i}+\delta V\right]\right)$ with $i \in\left[1 . . n_{s}\right]$. By this way, $10^{6}$ equi-possible realizations are generated.

3. For each random realization generated, the 3 characteristics $\left(V_{0}, n_{a}, P_{\text {veq }}\right)$ are identified using the procedure presented in the last section. This provides $10^{6}$ possible values of the equilibrium vapor pressure, $P_{\text {veq }}$.

4. Using the $R$ statistical numerical library (19), various normality tests are performed to ensure that the data set, $P_{v e q}$, is well-modeled by a Gaussian distribution with more than $99 \%$ confidence.

5. Finally, the uncertainty associated with this virtual experiment is estimated by $\Delta P_{v e q}= \pm 2 \sigma$ where $\sigma$ is the standard deviation. This guarantees that $95 \%$ of experiment values lie in this confidence interval.

Practically, a constant volume increment is used. Thus, the gas phase volume at the $i^{\text {th }}$ equilibrium stage can be written:

$$
V^{i}=i \times \Delta V \quad \text { with } \quad \Delta V=n_{r} \times V^{*}
$$

where $\Delta V$ is the volume increment imposed by the piston between two equilibrium situations. It depends on, $n_{r}$, the number of rotations done with the screw system and, $V^{*}$, the volume incre- 
ment for one rotation given in Table 1. Therefore, the computed global accuracy depends on 2 experimental choices, $n_{s}$, the number of equilibrium stages and, $n_{r}$, the number of rotations as defined above. Obviously, increasing these 2 quantities will improve the measure accuracy as clearly shown in Figure 3. These figures plot the equilibrium vapor pressure uncertainty, $\Delta P_{v e q}$, as a function of the number of equilibrium stages, $n_{s}$, on one hand, and of the number of rotations, $n_{r}$, on the other hand. In Figure $3 \mathrm{a}$, the number of rotations is imposed at $n_{r}=2$, while in Figure $3 \mathrm{~b}$ the number of equilibrium stages is fixed at $n_{s}=8$.
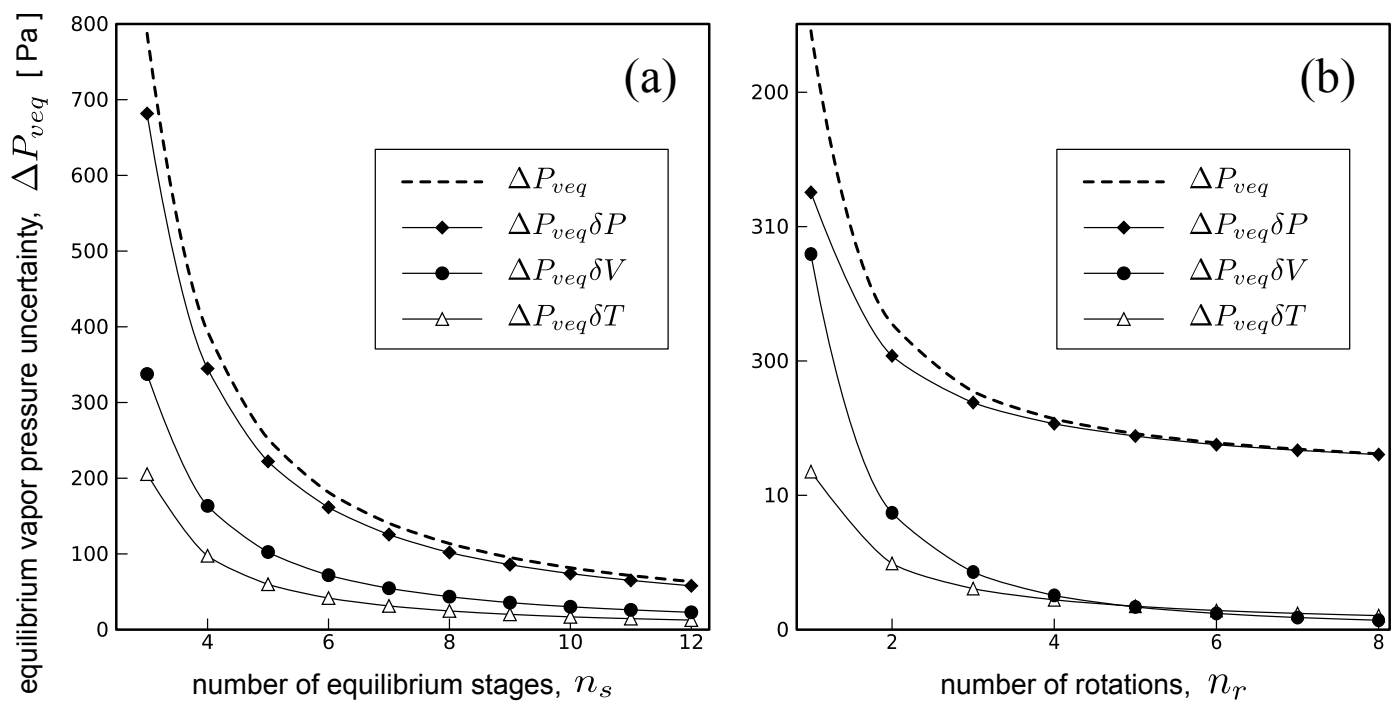

Figure 3: Total vapor pressure uncertainty, $\Delta P_{v e q}$, and uncertainties associated with each quantities, $\delta T, \delta P$ and $\delta V$ as functions of (a) the number of equilibrium stages, $n_{s}$, for $n_{r}=2-$ (b) the number of rotations, $n_{r}$, for $n_{s}=8$.

These figures also show the uncertainties, $\Delta P_{v e q}$, associated with each quantities, $\delta T, \delta P$ and $\delta V$, computed separately. For instance, $\Delta P_{v e q} \delta P$, is computed by taking $\delta T=0, \delta V=0$ and $\delta P=40 \mathrm{~Pa}$. Since the identification procedure is based on a pressure subtraction (Eq. (4)), these plots confirms that the pressure transducer accuracy is the most valuable characteristic, but also the most costly issue to improve.

Nevertheless, with a given experimental device, the total number of rotation, $n_{s} \times n_{r}$, cannot exceed a value associated with the screw length ((e) in Figure 1). With this upper limit fixed, similar numerical computations show that a better accuracy is obtained by increasing the equilibrium stages number, $n_{s}$, instead of the volume increment size, $n_{r}$. Eventually, an optimum should be 
found by using variable volume increments but this issue is beyond the scope of this paper.

\section{Results and Discussion}

\section{Validation of the method}

To experimentally validate the method, two approaches are presented. In the first one, we measure the vapor pressure regulated by standard saturated salt solutions. In the second one, we compare two techniques for determining the sorption isotherm of a reference soil.

The French National Standards Association (AFNOR) gives the relative humidity above various saturated salt solutions at different temperatures (NF X 15-119). They are used as reference materials to test the accuracy of our method. Therefore, validation tests have been carried out with 5 different saturated salt solutions that cover the whole range of water activity (Tab. 2). Each saturated salt solution is disposed inside the experimental device and the measures are conducted with a number of equilibrium stages, $n_{s}=8$, and a volume increment, $n_{r}=2$. The comparison between standards and experimental measures given in Tab. 2 asserts the accuracy of this technique over the whole range of water activity.

Table 2: Water activity for various saturated salt solutions at $30^{\circ} \mathrm{C}$ given by standards and measured with the activity-meter.

\begin{tabular}{lcc}
\hline Salt & $\begin{array}{c}\text { Water activity } \\
\text { from NF X 15-119 [/] }\end{array}$ & $\begin{array}{c}\text { Measured water activity } \\
{[/]}\end{array}$ \\
\hline Lithium chloride $\mathrm{LiCl}$ & $0.113 \pm 0.002$ & 0.1170 \\
\hline Magnesium chloride $\mathrm{MgCl}, 6 \mathrm{H}_{2} \mathrm{O}$ & $0.324 \pm 0.001$ & 0.3283 \\
\hline Magnesium nitrate $\mathrm{Mg}\left(\mathrm{NO}_{3}\right)_{2}, 6 \mathrm{H}_{2} \mathrm{O}$ & $0.520 \pm 0.001$ & 0.5213 \\
\hline Sodium chloride $\mathrm{NaCl}$ & $0.751 \pm 0.001$ & 0.7537 \\
\hline Potassium sulfate $\mathrm{K}_{2} \mathrm{SO}_{4}$ & $0.970 \pm 0.004$ & 0.9692 \\
\hline
\end{tabular}

The next validation test is based on a comparison with another method for determining the sorption isotherm of a reference soil using standard saturated salt solutions as described in the introduction. Samples are exposed to various constant-humidity atmospheres controlled by saturated salt solutions in a series of sealed chambers, all maintained at the same temperature. When 
equilibrium is reached, the moisture content is determined gravimetrically by weight loss or gain. Actually, if the samples are initially in a dry state, this procedure gives the adsorption isotherm while the desorption isotherm is obtained when porous samples are initially saturated.

With that objective, cylindrical soil samples (32-mm diameter and 15-mm high) have been used. The soil is a silty sand with a clayey fraction of $10 \%$ (20). It is oven-dried at $105^{\circ} \mathrm{C}$ for 24 hours. The required amounts of soil $\left(m_{s}=18.1 \mathrm{~g}\right)$ and demineralized water are added using a highprecision scale $\left(10^{-4} \mathrm{~g}\right)$. After mixing, it is stored in a airtight container for 24 hours to ensure the homogeneity of the water content. Then, the wet soil is compacted in a cylindrical ring by means of a hydraulic press in order to reach a dry density of $\rho_{s}=1500 \mathrm{~kg} \mathrm{~m}^{-3}$, which corresponds to a porosity of $\phi=43 \%$.

The couples $\left(w, a_{w}\right)$ obtained with both methods exposed above are reported in Figure 4. Much more experimental points are given with our method as it allows the entire range of water activity to be explored without being limited by the activity fixed by salt solutions. This aspect is particularly of great interest with high activity, $a_{w} \simeq 1$, since the upper limit of the hygroscopic domain is generally strenuously characterized $(18,21)$. Moreover, one measure can be done in about 4 hours what is considerably lower than the time required to reach an equilibrium above a salt solution.

One can note in Figure 4 that both curves are almost superimposed. There is a slight discrepancy since the curve obtained with this activity-meter is always below the one done with saturated salt solutions. Actually, both curves don't represent exactly the same physical quantity. The plot determined using salt solutions is a desorption isotherm curve as each experimental point are determined along a desorption path, i.e., samples initially at a large water content are subjected to a drying process to reach the water activity regulated by the salt solution. It is well-known that adsorption/desorption isotherm curves exhibit an hysteresis; the adsorption curve is generally below the desorption one. These two curves give the upper and lower limits in between every liquid-gas equilibrium state is. On the contrary, soil samples prepared to be characterized with the activitymeter have no sorption history. Therefore, the corresponding experimental points lie between adsorption and desorption state justifying the discrepancy revealed by Figure 4 . 


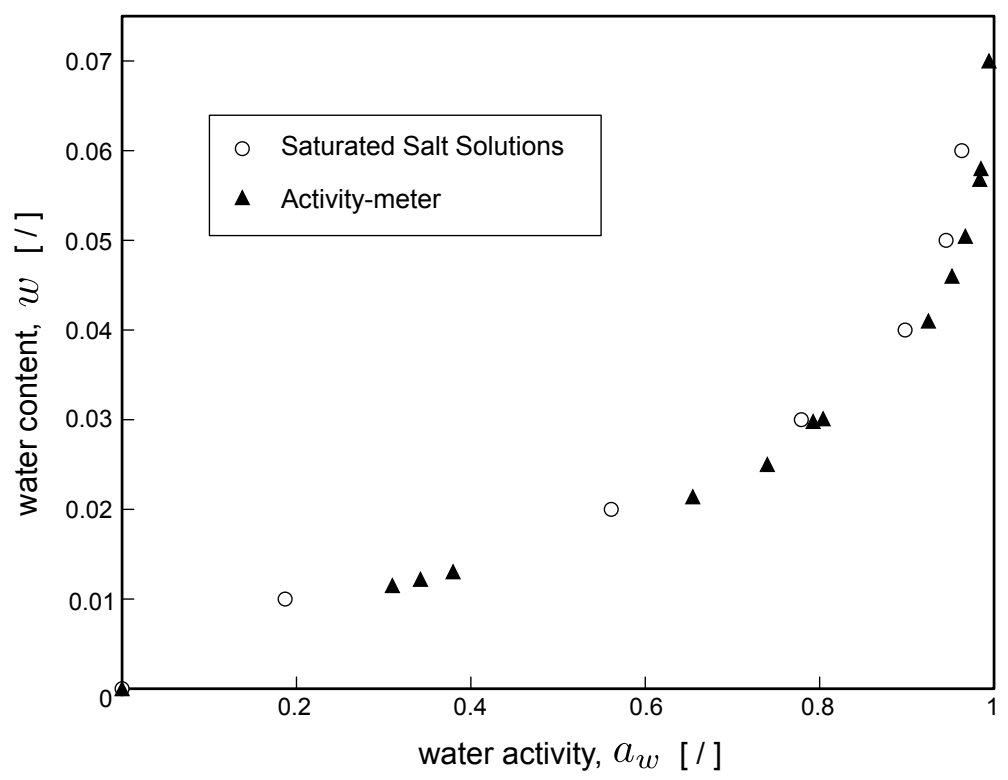

Figure 4: Comparison of sorption isotherm curves of water in a clayey silty sand determined using two different experimental methods.

\section{Application to various VOCs}

To emphasize the potentialities of this method, three liquid phases were used: water, trichloroethylene and heptane. The last two liquids are classical VOCs involved in many industrial soil contaminations. These three liquid phases are mixed with the soil following the same procedure described in previous section.

The sorption isotherms for these 3 compounds are presented in Figure 5. Beyond the classical isotherm obtained with water, this method allows us to determine the isotherm of any compound without calibration as long as its equilibrium vapor pressure is large enough to be measured with a pressure transducer. This clearly shows that hygroscopic effects are predominant at low liquid content not only with water, but also with any liquid phase. These sorption isotherms have been determined at oven-dried conditions, i.e., without moisture content. Several works have underlined the influence of moisture on vapor sorption of thrichloroethylene to soils $(22,23)$. Nevertheless, it has been suggested that the VOC sorption at any moisture content can be deduced from an sorption isotherm at oven-dried conditions (11). Anyway, these investigations are beyond the scope of this paper. 


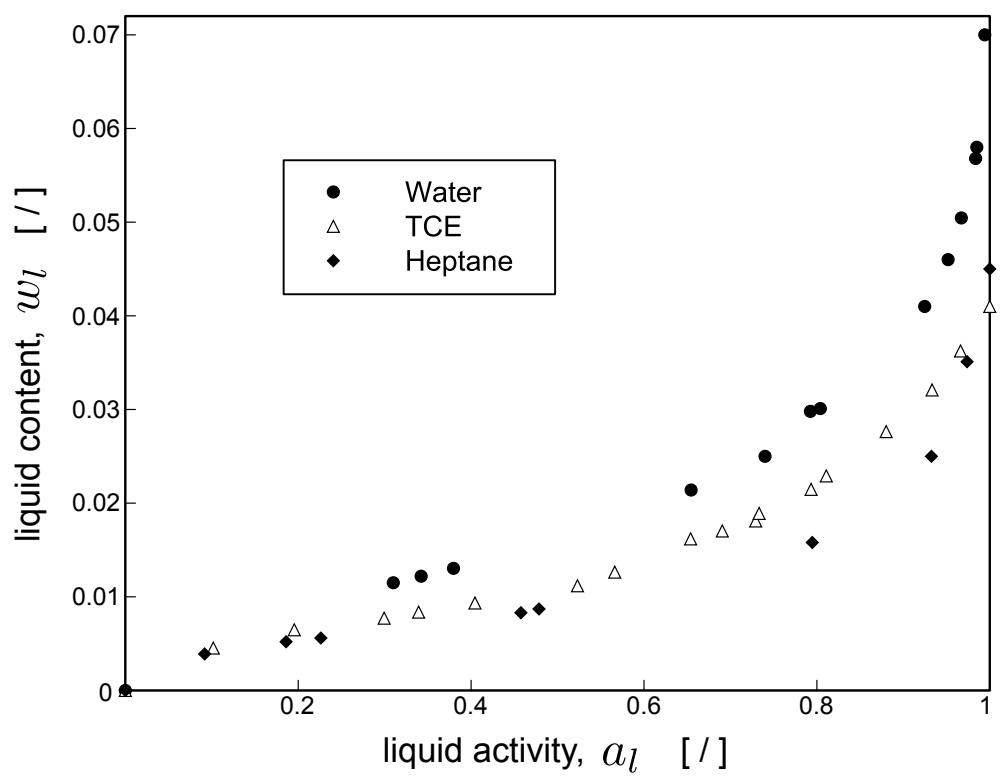

Figure 5: Sorption isotherms of water, TCE and heptane in a clayey silty sand determined with the activity-meter.

Moreover, the equilibrium vapor pressure obtained at large liquid content directly gives the saturated vapor pressure. Regarding to trichloroethylene and heptane, the saturated vapor pressures obtained in this work are compared successfully in Table 3 to references values from the literature $(24,25)$. Anyway, it should be noted that, according to the literature, the saturated vapor pressures are supposed to vary considerably. For instance considering TCE at $20^{\circ} \mathrm{C}$, references values range from $7700 \mathrm{~Pa}$ to $8600 \mathrm{~Pa}$.

Table 3: Saturated vapor pressures measured with the activity-meter compared to references values from the literature $(24,25)$

\begin{tabular}{lrrr}
\hline & temperature & $\begin{array}{r}\text { saturated vapor pressure } \\
\text { (activity-meter) }\end{array}$ & $\begin{array}{r}\text { saturated vapor pressure } \\
\text { (literature) }\end{array}$ \\
& {$[\mathrm{K}]$} & {$[\mathrm{Pa}]$} & {$[\mathrm{Pa}]$} \\
\hline Trichloroethylene & 293 & 7759 & 7601 \\
\hline & 303 & 11900 & 12220 \\
\hline Heptane & 303 & 7807 & 7726 \\
\hline
\end{tabular}




\section{Discussion}

The method presented here may have some similarities with the volumetric sorption technique used in surface area and pore size analyzes. The main difference is the use of volume variations instead of adding amount of vapor to modify the sorption equilibrium and thus the gas pressure. This slight difference has major consequences since it is really more simple to modify and control a gas volume rather than very small amount of vapor. Moreover, processing many equilibrium stages to determine one sorption equilibrium point leads to an accurate measurement even with common pressure transducers.

When compared to existing devices, this method presents several advantages. First of all, this method is easy to carry out and necessitates only classical and costless measurement sensors, a type-K thermocouple and a gas pressure transducer. The volume variations can be accurately imposed with a micrometric screw system allowing to automate the process. No chemical product or delicate sensor is required. Secondly, this procedure is versatile. Indeed, the accuracy is enhanced by using more equilibrium stages what also increases the measurement time. Therefore, with the same device, the experimental procedure can be adapted to conform to the desired accuracy. Finally, this method is usable with any volatile compound (VOC, hydrocarbon, solvent, ...) and is not restricted to water like standard protocols. Since the determination method does not depend on material or liquid characteristics, switching from a compound to another does not need any adjustment action or calibration procedure. Moreover, commercial devices based on a chilled mirror dewpoint sensor needs the knowledge of the complete saturated vapor pressure curve to relate the measured dewpoint temperature to the effective vapor partial pressure. This can be done with well-known compounds that have already been characterized. On the contrary, since our method directly determines the vapor partial pressure, no previous data is necessary. As shown in Table 3, the saturated vapor pressure curve of unknown organic compounds can be directly established with the apparatus presented here. 


\section{Acknowledgments}

This work was done within the framework of a collaboration with the university of Ouagadougou, Burkina-Faso and supported by the French Ministry of Foreign affairs.

\section{References}

(1) Gregg, S.; Sing, K. Adsorption, Surface Area and Porosity; Academic Press: New-york, 1967.

(2) Peterson, M.; Lion, L.; Shoemaker, C. Influence of vapor-phase sorption and diffusion on the fate of trichloroethylene in an unsaturated aquifer system. Environmental Science \& Technology 1988, 22, 571-578.

(3) Lin, T.-F.; Little, J.; Nazaroff, W. Transport and sorption of volatile organic compounds and water vapor within dry soil grains. Environmental Science \& Technology 1994, 28, 322-330.

(4) Werth, C.; Hansen, K. Modeling the effects of concentration history on the slow desorption of trichloroethene from a soil at $100 \%$ relative humidity. Journal of Contaminant Hydrology 2002, 54, 307-327.

(5) Shih, Y. Sorption kinetics and thermodynamics of trichloroethylene in humic acid. Colloids and Surfaces A: Physicochemical Engineering Aspects 2008, 317, 159-163.

(6) Tada, S.; Watanabe, K. Dynamic determination of sorption isotherm of cement based materials. Cement and Concrete Research 2005, 35, 2271-2277.

(7) Teoh, H.; Schmidt, S.; Day, G.; Faller, J. Investigation of cornmeal components using dynamic vapour sorption and differential scanning calorimetry. Journal of Food Science 2001, $66,434-440$.

(8) Campbell, C.; Campbell, G.; Cobos, D.; Carter, B.; Fontana, A. An Improved Method for Creating Moisture Release Curves in Dry Soils to Determine Mineralogical and Physical 
Properties. Joint Annual Meeting of the Soil Science Society of America, Oct 5-9, Houston, Texas, 2008.

(9) Hoff, J.; Gillham, R.; Mackay, D.; Shlu, W. Sorption of organic vapors at the air-water interface in a sandy aquifer material. Environmental Science \& Technology 1994, 27, 2789-2794.

(10) Steinberg, S.; Schmeltzer, J.; Kreamer, D. Sorption of Benzene and Trichloroethylene (TCE) on a Desert Soil: Effects of Moisture and Organic Matter. Chemosphere 1996, 33, 961-980.

(11) Unger, D.; Lam, T.; Schaefer, C.; Kosson, D. Predicting the effect of moisture on vapor-phase sorption of volatile organic compounds to soils. Environmental Science \& Technology 1996, 30, 1081-1091.

(12) Li, J.; Werth, C. Evaluating competitive sorption mechanisms of volatile organic compounds in soils and sediments using polymers and zeolites. Environmental Science \& Technology 2001, 35, 568-574.

(13) Gritti, F.; Guiochon, G. Accuracy and precision of adsorption isotherm parameters measured by dynamic HPLC methods. Journal of Chromatography A 2004, 1043, 159-170.

(14) Dette, S.; Ulrich, J. Comparison of Various Methods for Water Activity Determination. Chemical Engineering Technology 2006, 11, 1311-1315.

(15) Ouoba, S.; Cousin, B.; Cherblanc, F.; Bénet, J.-C. Une méthode mécanique pour mesurer la pression de vapeur d'équilibre de l'eau dans un milieu complexe. C. R. Mécanique 2010, 338, $113-119$.

(16) SciPy Reference Guide; Available at http://www.scipy.org/, 2010.

(17) Rahman, M. Food properties handbook; CRC Press: Boca Raton, Florida, 1995.

(18) Baucour, P.; Daudin, J. Development of a new method for fast measurement of water sorption isotherms in the high humidity range. Validation on gelatine gel. Journal of Food Engineering 2000, 44, 97-107. 
(19) R: A Language and Environment for Statistical Computing; Available at http://www.rproject.org/, 2009.

(20) Lozano, A.; Cherblanc, F.; Cousin, B.; Bénet, J.-C. Experimental study and modelling of the water phase change kinetics in soils. European Journal of Soil Science 2008, 59, 939-949.

(21) Rougier, T.; Bonazzi, C.; Daudin, J. Modeling incidence of lipid and sodium chloride contents on sorption curves of gelatin in the high humidity range. LWT - Food Science and Technology 2007, 40, 1798-1807.

(22) Chiou, C.; Shoup, T. Soil sorption of organic vapors and effects of humidity on sorptive mechanism and capacity. Environmental Science \& Technology 1985, 19, 1196-1200.

(23) Smith, J.; Chiou, C.; Kammer, J.; Kile, D. Effect of soil moisture on the sorption of trichloroethylene vapor to vadose-zone soil at Pictinny Arsenal, New Jersey. Environmental Science \& Technology 1990, 24, 676-683.

(24) Handbook of Chemistry and Physics, 90th ed.; Lide, D., Ed.; CRC Press: Boca Raton, Florida, 2009.

(25) Kirk-Othmer Encyclopedia of Chemical Technology, 5th ed.; Wiley-Interscience, John Wiley \& Sons: New York, 2007. 


\section{Brief}

An alternative method to characterize the sorption behavior of any liquid compound adsorbed in a soil is presented and applied to various VOCs. 